\title{
Temperature Distribution in InGaN-MQW LEDs under Operation
}

\author{
Veit Schwegler', Matthias Seyboth', Sven Schad ${ }^{1}$, Marcus Scherer, \\ Cristoph Kirchner ${ }^{1}$, Markus Kamp ${ }^{1}$, Ulrich Stempfle ${ }^{2}$, Wolfgang Limmer ${ }^{2}$, and \\ Rolf Sauer ${ }^{2}$ \\ ${ }^{1}$ Dept. of Optoelectronics, University of Ulm, 89069 Ulm, Germany \\ ${ }^{2}$ Dept. of Semiconductor Physics, University of Ulm, 89069 Ulm, Germany
}

\begin{abstract}
The temperature distribution in InGaN-MQW light emitting diodes was examined during operation with spatially resolved micro-Raman and micro-Electroluminescence measurements. The experimental results were compared to finite element simulations. A good agreement between the different experimental and calculated data is found.

Maximum operation temperatures up to $140{ }^{\circ} \mathrm{C}$ at a moderate forward currents of $30 \mathrm{~mA}$ are revealed by all three independent methods. Influences of substrate thickness, different substrates, and even bond-wires are shown.
\end{abstract}

\section{INTRODUCTION}

Generation of heat by ohmic losses limits performance and lifetime of lightemitting diodes (LEDs). Due to the high bandgap voltages GaN-based devices are subject of especially severe ohmic heating. Increased temperature reduces quantum efficiency and enhances diffusion of impurities (e.g. dopands, contact metals) as well as migration of dislocations [1].

UV-emitting InGaN-MQW LEDs, grown by low pressure MOVPE on sapphire substrates, are investigated regarding their junction temperature at different injection currents. Temperature distributions are determined by E2 phonon scattering (Raman), spatially resolved $\mu$-EL, and finite element simulations. Influences of substrate thickness (sapphire) and material (sapphire/SiC) on device temperatures are studied.

\section{EXPERIMENTAL}

Our UV-LEDs show narrow single peak emission at about $410 \mathrm{~nm}$. A buffer layer of $2 \mu \mathrm{m}$ undoped $\mathrm{GaN}$ is followed by $1 \mu \mathrm{m} \mathrm{Si}$-doped $\mathrm{GaN}$. The active region consists of a 4 x InGaN/GaN $(1.5 \mathrm{~nm} / 5.5 \mathrm{~nm})$ quantum well structure followed by a Mg-doped $\mathrm{Al}_{0.08} \mathrm{Ga}_{0.92} \mathrm{~N}$ electron barrier and $300 \mathrm{~nm}$ p-GaN cladding layer. In the $\mathrm{Mg}$-doped $\mathrm{GaN}$ we have free carrier concentrations of $\mathrm{p}=2-3 \times 10^{17} \mathrm{~cm}^{-3}$ in the Si-doped GaN of $\mathrm{n}=5 \mathrm{x}$ $10^{18} \mathrm{~cm}^{-3}$. A circular mesa is structured by chemically assisted ion beam etching. Contacts consist of an evaporated $\mathrm{Ni} / \mathrm{Au}$ metallization with an inner circular area as $\mathrm{p}$ contact and a surrounding $\mathrm{n}$-contact. These devices have series resistances of approximately $30 \Omega$. For measurements they are diced into pieces with a size of some $800 \mu \mathrm{m} \times 1800 \mu \mathrm{m}$ and mounted on teflon or metal heat sinks.

Operation of these LEDs on a temperature controlled heat sink displays impressively the problems aligned with temperature creation: Output power decreases exponentially with increased heat sink temperature (Figure. 1), since the internal quantum 


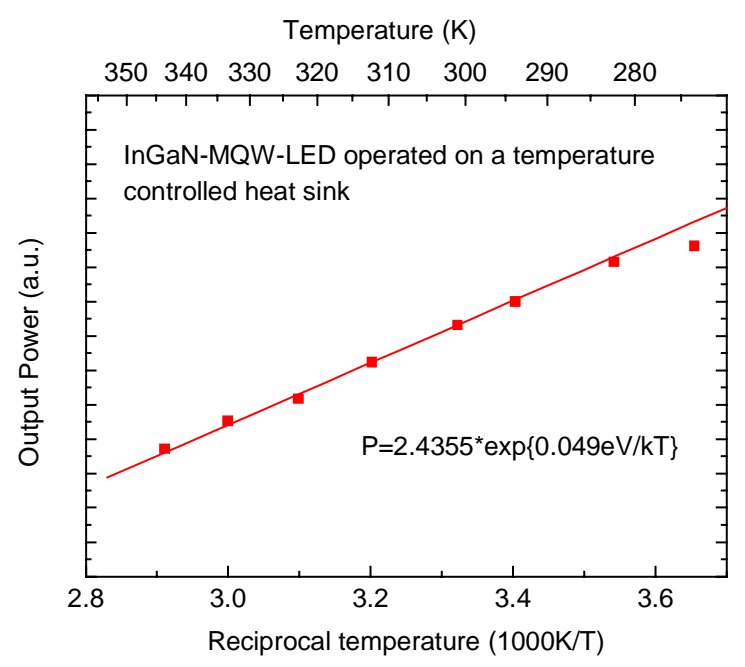

Figure 1: Temperature dependence of output power showing the almost exponential decrease of light output with increased temperature.

efficiency is depending almost exponentially on temperature according to equation (1) approximated for higher temperatures.

$$
\eta_{i}=\left(1+\left(\frac{\tau_{t} p N_{t}}{\tau_{r} n N_{r}}\right) \exp \left(-\frac{E_{t}-E_{r}}{k_{B} T}\right)\right)^{-1}
$$

with $\eta_{i}, \tau_{i}, n, p, N_{i}, E_{i}$ being the internal quantum efficiency, the lifetime, hole and electron concentration, concentrations and energies of radiative and non-radiative recombination mechanisms. The indices $t$ and $r$ account for trap and radiative related transitions.

The temperature of a LED can be derived from the emission spectrum with a fit using several fitting parameters [2], it is also possible to exploit only the short wavelength slope near the maximum of the EL spectrum for temperature determination according to reference [3].

Full fits on $\mu$-EL spectra are applied to get a spatially resolved picture of the temperature distribution. The backside emission of a LED was therefore spectrally and spatially mapped through the sapphire substrate. Figure 2 shows the integral EL intensity and wavelength distribution of a LED driven with a current density of $91 \mathrm{~A} / \mathrm{cm}^{2}$. The elevated current density beneath the p-contact is reflected by an increased temperature. A heat drain effect by the bondwire can be seen in a reduced redshift. Details are published in [4].

Temperature determination with Raman measurements makes use of the phonon shift. From the relative shift of the $\mathrm{GaN} \mathrm{E}_{2}$-phonon frequency of a LED under operational current with respect to the frequency at zero current, the temperature of the device can be 


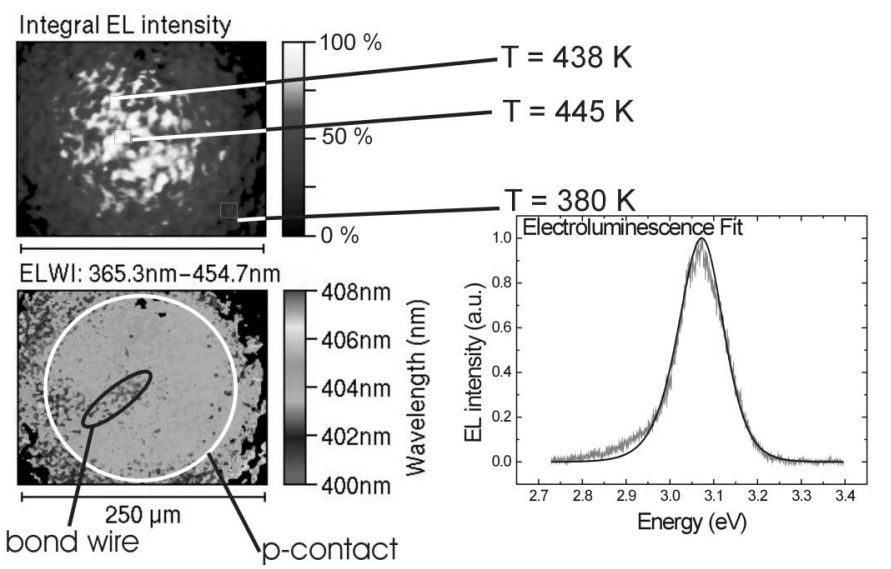

Figure 2. Temperature distribution derived at different points from $\mu$-EL (current density $j=320 \mathrm{~A} / \mathrm{cm}^{2}$ ), the redshift beneath the p-contact is due to increased temperature resulting from higher current density, the reduced redshift beneath the bond wire is due to heat drain effect. For an extended discussion of measuring method and results cf. [4]

computed. A detailed description of this measurement method is given in reference [5]. A spot diameter of $0.7 \mu \mathrm{m}$ permits high spatial resolution.

Results of Raman measurements are depicted in Figure 3 in comparison with data obtained from high energy slope fits of EL spectra. In good agreement, both methods reveal temperatures of about $415 \mathrm{~K}$ already at moderate currents of $30 \mathrm{~mA}$.

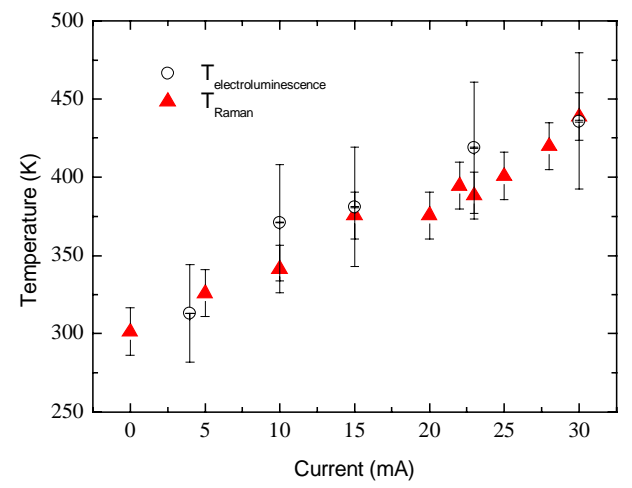

Figure 3. Temperature of LEDs under operation, LEDs mounted on teflon heat sink. Comparison of temperatures derived from EL and Raman measurements. In good agreement, both methods yield temperatures as high as $415 \mathrm{~K}$ even at moderate currents of $30 \mathrm{~mA}$.

F99W11.18 


\section{SIMULATION}

The finite element calculations for temperature distributions in LEDs are done using ANSYS, a well established commercial simulation program which is widely used for simulations of mechanical and electrical problems. Its capabilities include a combined thermal-electrical solver employing all linear effects and non-linear thermal transport properties. The simulations include the material parameters of $\mathrm{GaN}, \mathrm{InGaN}, \mathrm{AlGaN}$, $\mathrm{Al}_{2} \mathrm{O}_{3}, \mathrm{SiC}$ and $\mathrm{Ni} / \mathrm{Au}$ as contact materials. Temperature dependence of thermal conductivity, carrier concentration (particularly thermally depending $\mathrm{Mg}$ acceptor ionization), carrier mobility, and contribution to thermal conductivity from free carriers have been incorporated into the model. To account for our particular problem the geometry and electrical $I V$-characteristics of the LEDs are included. There are no fitting parameters within the simulation. Besides the mentioned material and device parameters the only input is the backside temperature serving as a boundary condition. This temperature has been measured with a thermal probe. The solver than returns a 3dimensional distribution (with radial symmetry) of electrical and thermal profiles present in the LEDs under various injection conditions as well as in dependence of the boundary conditions, i.e. thermal contact and properties of the base to which the LEDs are mounted. Figure 4 shows a cross section of such a simulated temperature distribution. Surface temperatures drawn of simulations were compared to Raman data (Figure 5). A refined simulation is improved by implementing heat drain by bond wires. A good match of both methods could be obtained.

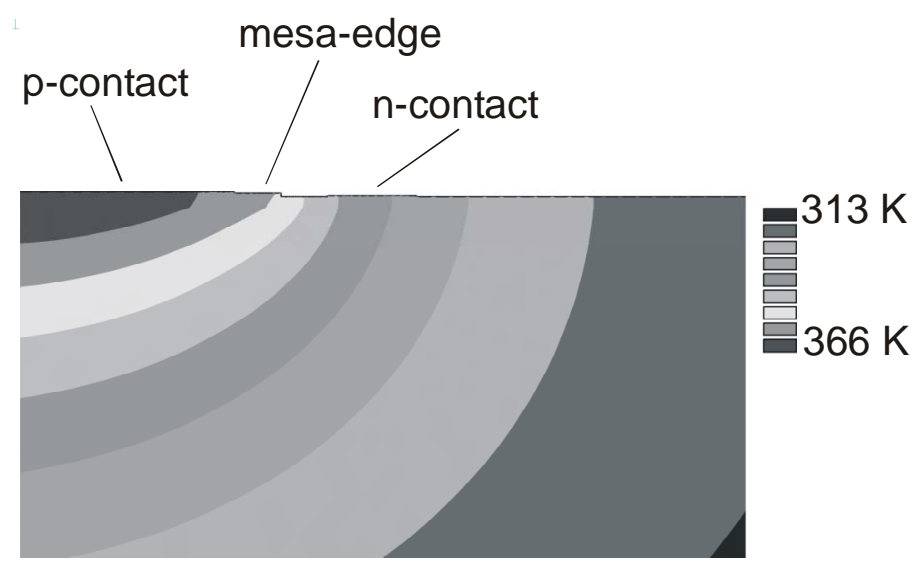

Figure 4. Cross section of temperature distribution in a LED under operational current of $63 \mathrm{~mA}$ mounted on metal heat sink obtained with finite element calculation. The elevated temperature beneath the p-contact is clearly visible. 


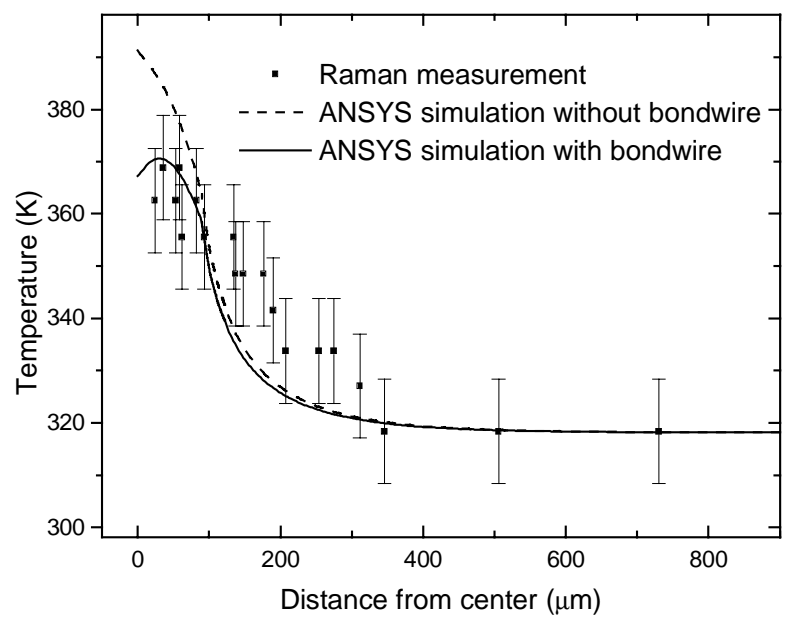

Figure 5. Comparison of spatially resolved temperature profiles obtained from Raman measurements and finite element calculations. Operation conditions as in Figure 4. The data show good agreement of both methods. The impact of a bond-wire on device temperature is demonstrated.

Considering the good agreement between simulation and experiment, we used ANSYS to calculate the influence of substrate thickness and material on temperatures in LEDs. Results are compiled in Figure 6. The simulations show strong impacts both of substrate thickness and material. Especially the improved heat transport through $\mathrm{SiC}$ substrates is clearly visible.
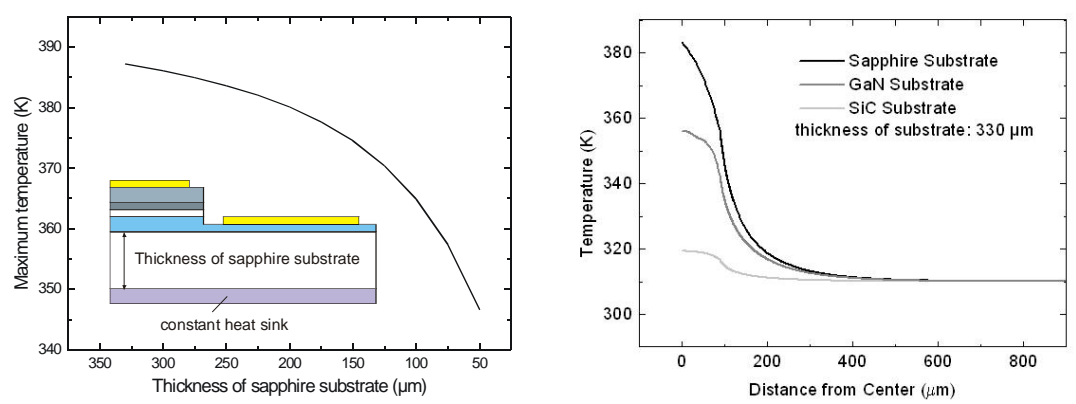

Figure 6. Device simulations with varied substrate thickness or material show the impact of the thermal conductivity of the substrate. Advantages of SiC are demonstrated. 


\section{CONCLUSIONS}

Using Raman and EL measurements together with finite element calculations we obtained a coherent picture on temperature distribution in InGaN-MQW LEDs. The different approaches reveal good agreement both in absolute temperature and in spatial distribution. The operating current causes significant heating limiting the output power and lifetime of devices. Even for moderate currents our devices show strong heating. Most heat is generated beneath the p-contact. Temperature drain effects of bond wires can be detected and simulated. The good agreement of simulation and experiment allows the employment of simulation as a versatile tool for further improvement of devices.

\section{ACKNOWLEDGEMENTS}

The authors would like to thank P. Fischer, J. Christen, and M. Zacharias (Univ. Magdeburg) for the $\mu$-EL measurements. Also we are grateful to A.E. Yunovich for the valuable discussions and assistance on fits of EL spectra. Financial support by German Ministry of Science and Education (BMBF) under Contract No. 01 BS 802 is gratefully acknowledged.

\section{REFERENCES}

1. L. Sugiura, J. Appl. Phys. 81, 1633 (1997).

2. K.G. Zolina, V. E. Kudryashov, A.N. Turkin, and A.E. Yunovich, Semiconductors 31, 901 (1997).

3. A.E. Yunovich, V.E. Kudryashov, A.N. Turkin, A.N. Kovalev, F.I. Manyakhin, MRS Internet J. Nitride Semic. Res. 4S1, G6.29 (1999).

4. P. Fischer, J. Christen, M. Zacharias, V. Schwegler, C. Kirchner, and M. Kamp, Appl. Phys. Lett. 75,. 3440 (1999).

5. A. Link, K. Bitzer, W. Limmer, R. Sauer, C. Kirchner, V. Schwegler, M. Kamp, D.G. Ebling, and K.W. Benz, J. Appl. Phys. 86, 6256 (1999). 